\title{
Crescimento de mudas de maracujazeiro-doce (Passiflora alata Curtis) associadas a fungos micorrízicos arbusculares (Glomeromycota) ${ }^{1}$
}

\author{
Maryluce Albuquerque da Silva², Uided Maaze Tibúrcio Cavalcante², Fábio Sérgio Barbosa da Silva², \\ Sueli Aparecida Gomes Soares² e Leonor Costa Maia ${ }^{2,3}$
}

Recebido em 22/08/2003. Aceito em 19/06/2004

\begin{abstract}
RESUMO - (Crescimento de mudas de maracujazeiro-doce (Passiflora alata Curtis) associadas a fungos micorrízicos arbusculares (Glomeromycota)). Para determinar o efeito de fungos micorrízicos arbusculares sobre o crescimento de mudas de maracujazeiro-doce foi conduzido, em casa de vegetação, experimento com delineamento inteiramente casualizado usando cinco tratamentos de inoculação (200 esporos/planta de Acaulospora longula Spain \& Schenck, Gigaspora albida Schenck \& Smith, Glomus etunicatum Becker \& Gerd., Scutellospora heterogama (Nicol. \& Gerd.) Walker \& Sanders e controle não inoculado), com sete repetições. A cada 20 dias foram avaliados altura e número de folhas e ao final do experimento (90 dias): diâmetro do caule, biomassa, área foliar, colonização micorrízica e densidade de esporos de FMA na rizosfera. A partir de 70 dias foram evidenciadas diferenças no número de folhas; aos 90 dias as plantas inoculadas com G. albida apresentaram maior altura que as demais, que não diferiram significativamente entre si. Plantas associadas com esse fungo apresentaram, em relação ao controle, incrementos de $2.138 \%$ e $1.430 \%$ nas biomassas fresca e seca da parte aérea, $1.937 \%$ na biomassa fresca da raiz e $2.671 \%$ na área foliar. Apesar de não existir especificidade de hospedeiro na associação micorrízica arbuscular, apenas G. albida promoveu respostas significativas no maracujazeiro-doce, indicando a existência de maior compatibilidade funcional entre esses simbiontes.
\end{abstract}

Palavras-chave: Pernambuco, micorriza, maracujazeiro-doce, Gigaspora albida, Scutellospora heterogama

\begin{abstract}
Growth of seedlings of sweet-passion fruit (Passiflora alata Curtis) associated to arbuscular mycorrhizal fungi (Glomeromycota)). In order to determine the effect of arbuscular mycorrhizal fungi (AMF) on growth of seedlings of sweet-yellow passion fruit, a greenhouse experiment was performed, using 200 AMF spores/plant in a randomized design with five treatments of inoculation (Acaulospora longula Spain \& Schenck, Gigaspora albida Schenck \& Smith, Glomus etunicatum Becker \& Gerd., Scutellospora heterogama (Nicol. \& Gerd.) Walker \& Sanders, and a non inoculated control) with seven replicates. Every 20 days height and leaf number and after 90 days shoot diameter, biomass, leaf area, root colonization and density of AMF spores in the rhizosphere were evaluated. After 70 days significant differences in number of leaves were observed. At the $90^{\text {th }}$ day plants associated with G. albida were higher than those of the other treatments that do not differ among them. In comparison with the control, plants associated with G. albida presented increment of $2,138 \%$ and $1,430 \%$ on fresh and dry biomass of the aerial part, 1,937\% on fresh biomass of roots and 2,671\% on leaf area. Although host specificity does not exist in arbuscular mycorrhizal associations, only G. albida promoted significant responses on growth of sweet-yellow passion fruit, what indicates that higher functional compatibility may exist between these partners.
\end{abstract}

Key words: Pernambuco State, mycorrhiza, sweet yellow passion fruit, Gigaspora albida, Scutellospora heterogama

\section{Introdução}

Os fungos micorrízicos arbusculares (FMA) são biotróficos obrigatórios que vivem em simbiose mutualista com raízes da maioria das plantas terrestres, promovendo maior absorção de nutrientes para o hospedeiro, que em troca cede ao fungo produtos da fotossíntese. Como característica da associação os fungos formam, no córtex da raiz hospedeira, estruturas especializadas (arbúsculos), responsáveis pela troca de nutrientes. $\mathrm{O}$ estabelecimento da simbiose micorrízica é determinado por fatores edafoclimáticos e aspectos da relação fungo-planta, com a utilização de diferentes espécies e/ou isolados de FMA resultando em respostas diferenciadas por parte do hospedeiro (Costa et al. 2001; Cavalcante et al. 2002).

Uma alternativa para o preparo de mudas de fruteiras é a utilização de FMA, os quais têm contribuído para diminuir o tempo para produção, reduzir a adubação fosfatada (Trindade et al. 2000; Cavalcante et al. 2001) e melhorar o pegamento das mudas, tornando-as mais capazes para suportar condições adversas no campo (Weber \& Amorim 1994).

\footnotetext{
1 Monografia da primeira Autora, bolsista de Iniciação Científica, CNPq

2 Departamento de Micologia, Centro de Ciências Biológicas, Universidade Federal de Pernambuco, CEP 50670-420, PE, Brasil

3 Autor para correspondência: leonorcmaia@yahoo.com.br
} 
Maracujazeiros com alta produtividade de frutos são obtidos a partir de mudas de qualidade, que em geral são produzidas com a adição de adubos orgânicos e/ou químicos (Ruggiero et al. 1996). Além desses insumos, a aplicação de FMA pode potencializar a produtividade desta fruteira, como observado por Cavalcante et al. (2002): a associação com Gigaspora albida Schenck \& Smith, Gigaspora margarita Becker \& Hall e Glomus etunicatum Becker \& Gerd., contribuiu para incrementar o desenvolvimento de mudas de maracujazeiro-amarelo reduzindo o tempo de produção.

O maracujazeiro-doce, apesar de produzir frutos ainda pouco disponíveis para venda e que atingem preços relativamente altos no mercado, constitui cultura rentável que vem crescendo nos últimos anos (Vasconcellos et al. 2001). No entanto, são necessárias pesquisas em todas as áreas, inclusive com relação à produção de mudas, visando o enquadramento da cultura como economicamente importante. Nesse contexto, a inoculação com FMA pode constituir importante ferramenta biotecnológica, para melhorar a adaptação, reduzir o estresse do transplantio e beneficiar o desenvolvimento de mudas desta fruteira.

Considerando que não há relatos sobre a simbiose micorrízica arbuscular em maracujazeiro-doce (Passiflora alata Curtis), foi conduzido bioensaio visando determinar o efeito desta associação na produção de mudas.

\section{Material e métodos}

O experimento, com duração de 90 dias a partir da inoculação das mudas com FMA, foi realizado em casa de vegetação, com delineamento inteiramente casualizado (DIC), sendo cinco tratamentos de inoculação (Acaulospora longula Spain \& Schenck (UFPE 21), Gigaspora albida (UFPE 01), Glomus etunicatum (UFPE 06), Scutellospora heterogama (Nicol. \& Gerd.) Walker \& Sanders (UFPE 19) e controle não inoculado), com sete repetições.

O solo utilizado, do tipo franco-arenoso, foi desinfestado com Bromex (98\% brometo de metila e $2 \%$ de cloropicrina) e apresentou as seguintes características: $8 \mathrm{mg} \mathrm{dm}^{-3}$ de P, 0,17; 3,75; $1,05 \mathrm{cmol}_{c} \mathrm{dm}^{-3}$ de $\mathrm{K}$, Ca, Mg, respectivamente, e pH 6,6.

Mudas uniformes de maracujazeiro doce foram obtidas a partir da germinação de sementes desinfestadas com hipoclorito de sódio $(0,05 \%$ por 2 minutos) e semeadas em bandejas de células contendo areia lavada e vermiculita ( $1: 1$ - v/v), esterilizadas em autoclave. Plântulas com duas folhas definitivas foram transferidas para recipientes plásticos de $300 \mathrm{~mL}$, contendo $280 \mathrm{~mL}$ de solo onde se adicionou, na região da raiz, solo-inóculo contendo cerca de 200 esporos dos FMA mencionados; após 15 dias as plântulas foram transferidas para sacos de polietileno preto contendo 1,6kg de solo, mantendo-se uma plântula/saco.

A cada vinte dias após a inoculação, foram tomadas medidas de altura e número de folhas. Após a colheita (90 dias), foram avaliados: diâmetro do caule, área foliar, biomassa fresca e seca da parte aérea, biomassa fresca das raízes, colonização das raízes e densidade de esporos de FMA na rizosfera.

Amostras de 0,5g de raízes de cada tratamento foram lavadas, clarificadas com $\mathrm{KOH}(10 \%)$, coradas com azul de Trypan (Phillips \& Hayman 1970) e avaliadas quanto à percentagem de colonização micorrízica (Giovannetti \& Mosse 1980). A parte aérea das plantas foi colocada em estufa de circulação de ar $\left(65^{\circ} \mathrm{C}\right)$ até peso constante, para determinação da biomassa seca.

A determinação da densidade de esporos do soloinóculo e a contagem final dos esporos no solo após a colheita do experimento foram feitas utilizando alíquotas de $50 \mathrm{~g}$ de solo e extração por peneiramento úmido (Gerdemann \& Nicolson 1963) e centrifugação em água e sacarose 40\% (Jenkins 1964). Os esporos foram recolhidos e colocados em placa canaletada, para contagem em microscópio estereoscópico (40×).

Os dados foram submetidos à análise de variância e as médias comparadas pelo teste de Tukey $(\mathrm{P} \leq 0,05)$, utilizando o programa Statistica (StatSoft 1997).

\section{Resultados e discussão}

Noventa dias após a inoculação, mudas inoculadas com G. albida apresentaram maior crescimento (altura, número de folhas, diâmetro do caule, biomassa fresca da parte aérea e da raiz, biomassa seca da parte aérea e área foliar) do que as demais, inoculadas ou não com FMA (Tab. 1). Em todos os parâmetros foi observado que as mudas em associação com A. longula, G. etunicatum e S. heterogama apresentaram crescimento similar ao controle não micorrizado. $\mathrm{O}$ incremento no crescimento das mudas, proporcionado por G. albida, alcançou $2.138 \%$ e $1.430 \%$, respectivamente nas biomassas fresca e seca da parte aérea, $1.937 \%$ na biomassa fresca da raiz e $2.671 \%$ na área foliar, em relação ao controle não inoculado. Adicionalmente, o diâmetro do caule de plantas inoculadas com G. albida foi significativamente maior 
Tabela 1. Dados de crescimento de mudas de maracujazeiro-doce (Passiflora alata Curtis) e de fungos micorrízicos arbusculares, 90 dias após a inoculação.

\begin{tabular}{|c|c|c|c|c|c|c|c|c|c|}
\hline \multirow[t]{2}{*}{ Tratamentos } & \multicolumn{9}{|c|}{ Parâmetros avaliados } \\
\hline & $\begin{array}{c}\mathrm{A} \\
(\mathrm{cm})\end{array}$ & NF & $\begin{array}{l}\mathrm{DM} \\
(\mathrm{mm})\end{array}$ & $\begin{array}{l}\text { BFA } \\
\text { (g) }\end{array}$ & $\begin{array}{c}\text { BFR } \\
\text { (g) }\end{array}$ & $\begin{array}{c}\text { BSA } \\
\text { (g) }\end{array}$ & $\begin{array}{c}\mathrm{AF} \\
\left(\mathrm{cm}^{2}\right)\end{array}$ & $\begin{array}{c}\mathrm{C} \\
(\%)\end{array}$ & $\begin{array}{c}\mathrm{DE} \\
\text { (n./g solo) }\end{array}$ \\
\hline Gigaspora albida & 10,68 a & $12,77 \mathrm{a}$ & $0,250 \mathrm{a}$ & $6,94 \mathrm{a}$ & 4,89 a & $1,53 \mathrm{a}$ & 263,61 a & 64,0 a & $1,34 \mathrm{a}$ \\
\hline Scutellospora heterogama & $5,20 \mathrm{~b}$ & $4,60 \mathrm{~b}$ & $0,136 \mathrm{~b}$ & $0,76 \mathrm{~b}$ & $0,47 \mathrm{~b}$ & $0,13 \mathrm{~b}$ & $25,89 \mathrm{~b}$ & $22,5 b$ & $0,12 \mathrm{~b}$ \\
\hline Acaulospora longula & $4,38 \mathrm{~b}$ & $2,00 \mathrm{~b}$ & $0,142 \mathrm{~b}$ & $0,28 \mathrm{~b}$ & $0,31 \mathrm{~b}$ & $0,09 \mathrm{~b}$ & $6,17 \mathrm{~b}$ & $26,8 \mathrm{~b}$ & $0,04 \mathrm{~b}$ \\
\hline Glomus etunicatum & $4,58 \mathrm{~b}$ & $1,57 \mathrm{~b}$ & $0,131 \mathrm{~b}$ & $0,25 \mathrm{~b}$ & $0,30 \mathrm{~b}$ & $0,09 \mathrm{~b}$ & $7,97 \mathrm{~b}$ & $18,2 \mathrm{~b}$ & $0,12 \mathrm{~b}$ \\
\hline Controle & $4,41 \mathrm{~b}$ & $1,50 \mathrm{~b}$ & $0,138 \mathrm{~b}$ & $0,31 \mathrm{~b}$ & $0,24 \mathrm{~b}$ & $0,10 \mathrm{~b}$ & $9,51 \mathrm{~b}$ & $0,0 \mathrm{c}$ & $0,00 \mathrm{~b}$ \\
\hline
\end{tabular}

A - altura; NF - número de folhas; DM - diâmetro do caule; BFA - biomassa fresca da parte aérea; BFR - biomassa fresca da raiz; BSA - biomassa seca da parte aérea; AF - área foliar; C - colonização; DE - densidade de esporos de FMA na rizosfera. Dados seguidos da mesma letra, na coluna, não diferem pelo teste de Tukey $(\mathrm{P}<0,05)$.

do que o observado nos demais tratamentos.

Diferenças significativas no número de folhas foram evidenciadas a partir dos 50 dias no tratamento com G. albida, em relação ao controle e G. etunicatum, e aos 70 e 90 dias entre G. albida e os demais tratamentos (Fig. 1). Para A. longula, G. etunicatum e o controle, as diferenças foram relacionadas com a perda de folhas durante o experimento, principalmente aos 90 dias. Isso pode ter ocorrido devido à condição de baixa fertilidade do solo, pois nestes tratamentos as folhas apresentavam clorose indicando deficiência nutricional. As mudas associadas a $S$. heterogama, que tinham perdido folhas aos 70 dias, apresentaram tendência para recuperação aos 90 dias, indicando efeito tardio deste fungo conforme observado também por Cavalcante et al. (2002) em plantas de maracujazeiro-amarelo (Passiflora edulis Sims. f. flavicarpa Deg.) após 70 dias da inoculação

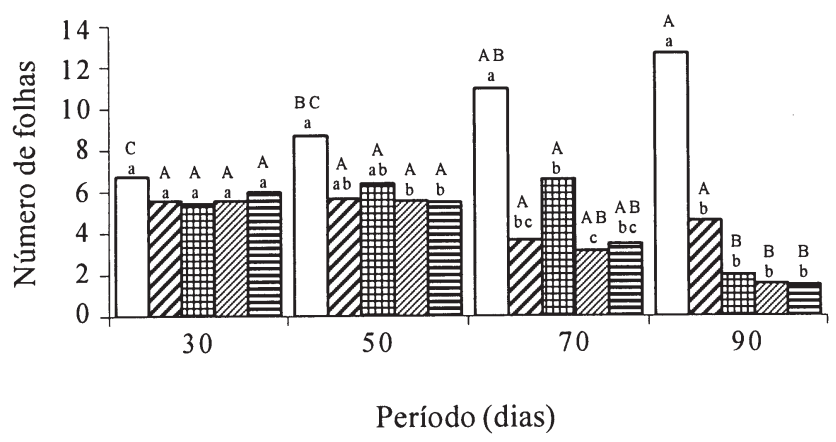

Figura 1. Número de folhas de plantas de maracujazeiro-doce (Passiflora alata Curtis) associadas ou não a FMA, após 30, 50, 70 e 90 dias em casa de vegetação. Dados nas colunas seguidas da mesma letra minúscula, dentro de cada período e maiúscula, no mesmo tratamento de inoculação entre os períodos, não diferem estatisticamente $(\mathrm{P}<0,05) . \square=$ G. albida; $\square=S$. heterogama; 四=A. longula; 四 = G. etunicatum; 目= Controle. com o mesmo isolado de FMA. Quanto à altura, diferenças foram observadas apenas aos 90 dias, sendo as mudas inoculadas com G. albida maiores que as demais, que apresentaram tamanhos similares (Fig. 2).

Alguns autores relataram o beneficio de várias culturas, quando associadas a espécies de Gigaspora. Em maracujazeiro-amarelo, após 50 dias, a inoculação com G. albida e G. margarita beneficiou o crescimento das mudas, o que não ocorreu na associação com $S$. heterogama e A. longula (Cavalcante et al. 2002). Neste experimento, aumento no crescimento do maracujazeiro-doce só ocorreu em mudas associadas a G. albida. Respostas similares foram obtidas em gravioleiras (Annona muricata L.) (Chu et al. 2001) e mamoeiros (Carica papaya L.) (Trindade et al. 2001) associados a G. margarita.

Embora os FMA não possuam especificidade hospedeira, há associações preferenciais entre espécies

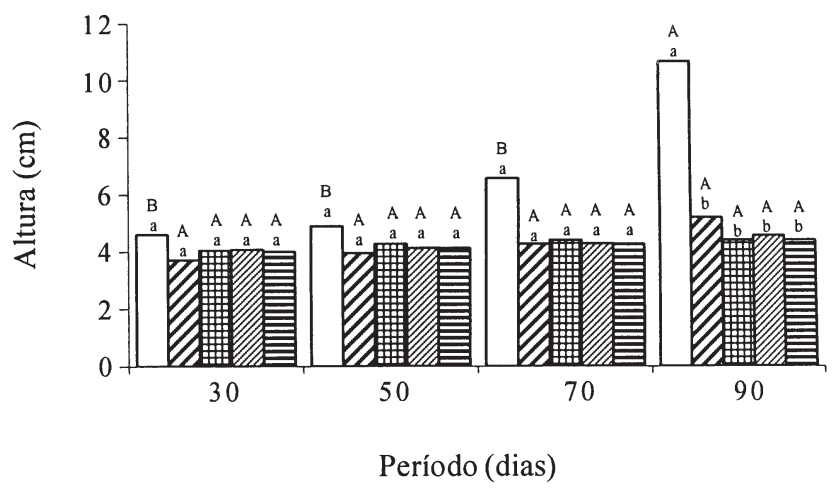

Figura 2. Altura de plantas de maracujazeiro-doce (Passiflora alata Curtis) associadas ou não a FMA, após 30, 50, 70 e 90 dias em casa de vegetação. Dados nas colunas seguidas da mesma letra minúscula, dentro de cada período e maiúscula, no mesmo tratamento de inoculação entre os períodos, não diferem estatisticamente $(\mathrm{P}<0,05) . \square=$ G. albida; $\square=S$. heterogama; 囲 =A. longula $;=$ G. etunicatum; 目 $=$ Controle. 
vegetais e determinados isolados de FMA, que podem refletir no crescimento do hospedeiro associado, bem como na colonização e esporulação dos fungos apesar de não haver, necessariamente, correlação entre esses parâmetros (Silveira 1998).

Os genótipos do fungo e da planta influenciam a colonização e o desempenho da simbiose (Silveira 1998), como demonstrado em experimentos com aceroleiras (Malpighia emarginata D.C.): mudas do genótipo Barbados apresentaram melhor resposta de crescimento quando inoculadas com G. margarita, enquanto as do genótipo Miró foram beneficiadas pela associação com G. etunicatum (Costa et al. 2001).

O tipo do substrato também pode influenciar a simbiose principalmente pela quantidade de nutrientes disponíveis. Silva et al. (2001) verificaram que mudas de maracujazeiro-amarelo cultivadas em vermiculita e inoculadas com FMA apresentaram melhor crescimento do que as cultivados em substrato rico em nutrientes (Plantmax ${ }^{\circledR}$ ). Em estudo com mamoeiros, a resposta à micorrização foi mais acentuada quando as plantas foram submetidas a doses de 12 a $16 \mathrm{mg} \mathrm{dm}^{-3} \mathrm{de} P$ disponível (Trindade et al. 2001). No caso do maracujazeiro-amarelo, o crescimento foi favorecido pela associação com FMA (G. albida e S. heterogama) em solos com 4 e $11 \mathrm{mg} \mathrm{dm}^{-3}$ de $\mathrm{P}$, sendo essa cultura extremamente dependente da micorrização quando em solo com o menor teor de P (Cavalcante et al. 2001). No presente trabalho, além da melhor interação do maracujazeiro-doce com G. albida, a quantidade de $\mathrm{P}$ no solo $\left(8 \mathrm{mg} \mathrm{dm}^{-3}\right)$ pode ter favorecido a associação, considerando que a simbiose se estabelece melhor quando em substrato com baixos níveis de P (Saggin-Júnior \& Siqueira 1996).

Embora colonizadas, plântulas inoculadas com G. etunicatum, A. longula e S. heterogama tiveram crescimento lento e, ao serem colhidas 90 dias após a inoculação, não haviam atingido a altura adequada para o transplantio ao campo, do mesmo modo que as plantas controle, que não apresentaram colonização. As taxas de colonização proporcionadas por esses FMA podem ser consideradas baixas $(<30 \%)$, sobretudo quando comparadas com a promovida por G. albida (> 60\%), que também apresentou maior esporulação (Tab. 1).

Considerando que o crescimento do maracujazeirodoce foi incrementado apenas pela associação com G. albida, pode-se atribuir o beneficio da simbiose à combinação FMA $\times$ hospedeiro, visto que as demais condições do experimento foram iguais para todos os tratamentos. Assim, apesar de supostamente não existir especificidade pelo hospedeiro na simbiose micorrízica arbuscular, os resultados indicam a existência de maior compatibilidade funcional entre o maracujazeiro-doce e o isolado de G. albida, do que entre essa fruteira e os demais fungos utilizados. Desse modo, a associação micorrízica pode favorecer o crescimento do maracujazeiro-doce, desde que seja aplicado um isolado de FMA compatível.

\section{Agradecimentos}

Ao CNPq, pelo apoio financeiro.

\section{Referências bibliográficas}

Cavalcante, U.M.T.; Maia, L.C.; Costa, C.M.C.; Cavalcante A.T. \& Santos, V.F. 2002. Efeito de fungos micorrízicos arbusculares, da adubação fosfatada e da esterilização do solo no crescimento de mudas de maracujazeiro amarelo. Revista Brasileira de Ciências do Solo 26: 1099-1106.

Cavalcante, U.M.T.; Maia, L.C.; Costa, C.M.C. \& Santos, V.F. 2001. Mycorrhizal dependency of passion fruit (Passiflora edulis f. flavicarpa). Fruit 56: 317-324.

Cavalcante, U.M.T.; Maia, L.C.; Melo, A.M.M. \& Santos, V.F. 2002. Influência da densidade de fungos micorrízicos arbusculares na produção de mudas de maracujazeiroamarelo. Pesquisa Agropecuária Brasileira 37: 643-649.

Chu, E.Y.; Moller, M.R.F. \& Carvalho, J.G. 2001. Efeitos da inoculação micorrízica em mudas de gravioleira em solo fumigado e não fumigado. Pesquisa Agropecuária Brasileira 36(4): 671-680.

Costa, C.M.C.; Maia, L.C.; Cavalcante, U.M.T. \& Nogueira, R.J.M.C. 2001. Influência de fungos micorrízicos arbusculares sobre o crescimento de dois genótipos de aceroleira (Malpighia emarginata D.C.). Pesquisa Agropecuária Brasileira 36: 893-901.

Gerdeman, J.W. \& Nicolson, T.H. 1963. Spores of mycorrhizal Endogone species extracted from soil by wet sieving and decanting. Transactions of the British Mycological Society 46: 235-244.

Giovannetti, M. \& Mosse, B. 1980. An evaluation of techniques for measuring vesicular arbuscular mycorrhizal infection in roots. New Phytologist 84: 489-500.

Jenkins, W.R. 1964. A rapid centrifugal-flotation technique for separating nematodes from soil. Plant Disease Report 48: 692.

Phillips, J.M. \& Hayman, D.S. 1970. Improved procedures for clearing roots and staining parasitic and vesicular arbuscular mycorrhizal fungi for rapid assessment of infection. Transactions of the British Mycological Society 55: 157-161.

Ruggiero, C.; São José, A.R.; Volpe, C.A.; Oliveira, J.C.; Durigan, J.F.; Baumgartner, J.G.; Silva, J.R.; Nakamura, K.; Ferreira, M.E.; Kavati, R. \& Pereira V.P. 1996. Maracujá para exportação: aspectos técnicos da produção. Brasília, Embrapa-SPI, 64p. (FRUPEX, 19). 
Saggin-Júnior, O.J. \& Siqueira, J.O. 1996. Micorrizas arbusculares em cafeeiro. Pp. 203-254. In: J.O. Siqueira (ed.). Avanços em fundamentos e aplicação de micorrizas. Lavras, Universidade Federal de Lavras.

Silva, R.P.; Peixoto, J.R. \& Junqueira, N.T.V. 2001. Influência de diversos substratos no desenvolvimento de mudas de maracujazeiro azedo (Passiflora edulis Sims. f. flavicarpa Deg.). Revista Brasileira de Fruticultura 23: 377-381.

Silveira, A.P.D. 1998. Micorrizas. Pp. 61-86. In: I.S. Melo \& J.L. Azevedo (eds.). Ecologia Microbiana. Jaguariúna, Embrapa.

StatSoft. 1997. Statistic for Windows 5.1. CD ROM. Tulsa, StatSoft Inc.
Trindade, A.V.; Siqueira, J.O. \& Almeida, F.P. 2001. Dependência micorrízica de variedades comerciais de mamoeiro. Pesquisa Agropecuária Brasileira 36: 1485-1494.

Trindade, A.V.; Siqueira, J.O. \& Almeida, F.P. 2000. Eficiência simbiótica de fungos micorrízicos arbusculares em solo não fumigado para mamoeiro. Revista Brasileira de Ciência do Solo 24: 505-513.

Vasconcellos, M.A.S.; Brandão Filho, J.U.T. \& Vieites, R.L. 2001. Maracujá-doce. Pp. 387-416. In: C.H. Bruckner \& M.C. Picanço (eds.). Maracujá: tecnologia de produção, pós-colheita, agroindústria, mercado. 20 ed., Porto Alegre, Editora Cinco Continentes.

Weber, O.B \& Amorim, S.M.C. 1994. Adubação fosfática e inoculação de fungos micorrízicos vesículo-arbusculares em mamoeiro "solo". Revista Brasileira de Ciência do Solo 18: 187-191. 